O.P. NARIEZHNII, Cand. Sc. (Technology), T.O. GRINENKO, Cand. Sc. (Technology), I.D. GORBENKO, Dr. Sc. (Technology)

\title{
STATEMENT OF THE PROBLEM OF ASSESSING INSTABILITY OF PASSIVE QUANTUM FREQUENCY STANDARDS IN THE PRESENCE OF AN ERROR FROM THE INTERACTION
}

\section{Introduction}

Problem statement. Creation of quantum generators (masers and lasers) is deservedly considered one of the greatest achievements of physics in the second half of the 20th century [1]. This discovery led to the emergence of a new branch of technical physics, namely, quantum electronics. In this area of outwardly traditional research, the issues of the theory of interaction of a radio-frequency field with matter and the elements of the theory of quantum amplifiers and generators have been sufficiently well studied. However, over the past two decades, revolutionary changes have taken place that have significantly transformed the scientific and technological appearance of quantum electronics. These changes are associated, first of all, with the emergence of many new fundamental and applied problems of coordinate-time support [2-4]. As a result of such a radical expansion of the range of problems, the issues of the influence of the error from the interaction on the estimation of the frequency instability of passive quantum standards of frequency (QSF) are still poorly understood. This is due to the laboriousness of this measuring task associated with the involvement of complex and expensive equipment. This equipment makes it possible to use the atomic time scales (TS), formed by global navigation satellite systems (GNSS) such as GPS IGLONASS, and atomic weighted average scales of spatially separated group standards of frequency and time.

Analysis of the literature. Of the whole variety of studied atomic and molecular transitions caused by hyperfine interactions in atoms or the result of perturbations of the electronic structure in molecules, to create frequency references, atoms or molecules are mainly used, the transition frequencies of which lie in the range of $1 \ldots 30 \mathrm{GHz}$ [5 - 7]. At the same time, the following types of the QSF have been studied quite well: quantum generators based on beams of ammonia molecules, beams of hydrogen atoms and rubidium vapor with optical pumping; passive QSF on beams of cesium atoms and rubidium vapor with optical pumping and optical indication, etc. [5, 8].

The functioning of these QSF is based on the methods of radio spectroscopy. For example, the most common cesium and rubidium QSF are based on the method of passive atomic beams, based on the interaction of a radio-frequency field with a beam of atoms or molecules. Because of interaction, transitions occur between atomic states. The most precise hydrogen QSF, as well as optically pumped rubidium standards, are based on the method of constructing a standard on a maser, where an atom or molecule is injected into a resonator tuned to the transition frequency.

The accuracy of the passive QSF, based on the measurement of the position of the resonance absorption line, depends on the width of the spectral line. The narrower the line, the higher the accuracy. It is known, that the effect of external fluctuating fields of different physical nature (temperature, electromagnetic, gravitational, etc.) on the QSF leads to the appearance of additional frequency fluctuations in their output signal, due to these influences. A large number of specialists in the theory of quantum amplifiers and oscillators are engaged in compensation for these destabilizing factors affecting the spectral line width.

In general, the issues of the QSF interaction when combining their group have not been sufficiently studied to date. The issues of electromagnetic compatibility of frequency measures in a group are especially poorly studied. In this case, the standard frequency separation requirement does not apply, since all frequency measures used have practically the same frequency of oscillation. 


\section{The main part}

Determination of spatial coordinates and velocity components is based in navigation systems on rangefinder and Doppler measurements. At the same time, the need for high stability of the systematic TS increases as the requirements for the accuracy of navigation determinations increase, especially when using the passive GNSS rangefinder method such as GPSIGLONASS. Therefore, the radio navigation field in the GNSS data, along with the main function (global autonomous operational navigation of ground mobile objects), allows mutual synchronization of the QSF at remote ground objects. The most precise comparisons of the TS using GNSS are carried out in the differential navigation mode. The differential navigation method is based on the relative constancy of a significant part of the GNSS errors in time and space [3].

In navigation systems, the formation of the systemic TS and its maintenance (storage) during the entire life of the system is carried out by atomic clocks. For modern atomic clocks, the relative frequency instability is $(1 \ldots 5) \cdot 10^{-14}$ and below [7]. Of course, to maintain such a high stability, it is necessary to create a complex hardware complex. The task of this complex is to ensure the functioning of the core of the atomic clock, namely, an atomic (quantum) frequency standard under conditions of constant temperature, minimal influence of external and internal electromagnetic fields, exclusion of vibrations, etc.

It is known that the equation of the atomic clock motion, based on the fundamental concepts of quantum mechanics, in the general case can be represented in the $j \frac{h}{2 \pi} \partial \Psi / \partial t=\mathrm{H} \Psi$ form, where $\Psi$ is the wave function; $\mathrm{H}$ is the Hamiltonian; $h$ is Planck's constant, and $j=\sqrt{-1}$. The consequence of this equation of motion of the atomic clock (QSF) is the law $\Delta E=h v$, where $\Delta E$ the difference in energy is, $v$ is the frequency of radiation. Thus, the postulate of the high stability of the atomic TS follows from the assumption of the invariability of the frequency corresponding to the energy difference $\Delta E$.

At the same time, despite significant advances in the creation of atomic clocks (QSF), there are areas of their practical application, which, in principle, cannot be satisfied with the achieved level. These areas include metrological support for time and frequency measurements. In some cases, while ensuring high metrological characteristics, extremely high requirements are imposed on the reliability of the device.

These difficulties are often solved in practice by building group time and frequency keepers. Analytical (based on calculations) and instrumental (based on appropriate signal conversion) combining of the QSF (generators) into one group, as stated in [9-16], makes it possible to increase the accuracy and stability of the keeper based on averaging the characteristics of several generators and reliability based on their reservation. Modern group time and frequency keepers include up to ten, and reference means up to several dozen QFS.

Analytical methods of averaging the frequency of group keepers allow calculating corrections to the frequency of individual custodians at any time. The use of these corrections in order to regulate the frequencies of the keepers allows maintaining the frequency at the output of the keeper close to the weighted average. The weight (contribution) of each QSF from the group to the formation of the weighted average frequency is inversely proportional to the estimate of the variance of the frequency deviation of each of them from the weighted average for the entire group [11].

Determination of weighting factors based on the results of intercomparisons of QSF in a group encounters two problems.

First, to obtain unbiased estimates of the weighting coefficients, it is necessary to estimate and eliminate the average value of deviation of the frequency of each QSF from the weighted average value. This value, in turn, is determined from the condition that the unbiased estimates of the weight coefficients are known. That is, a certain contradiction arises, the resolution of which allows inaccuracies in the form of certain assumptions based on subjective factors. 
Secondly, since only the frequency differences of the output signals of the used QSF can be determined in the process of intercomparisons, in the estimates of the variance of the results of paired comparisons, in addition to the estimates of the variance of the intrinsic noise of each measure, second mixed central moments are added. In the general case, in the presence of an error from mutual interaction, for a system of $N$ measures, there is a need to determine $N^{2}$ unknown estimates of the variance of the intrinsic noise of each quantum measure (QSF) and the second mixed central moments. When implementing a complete graph of comparisons, it is possible to obtain $(N-1)^{2}$ estimates of the second central moments, which can be used as the right-hand sides of a system of linear equations with respect to the desired quantities. According to the theorems of linear algebra, such a system has no unique solution. This determines the inability to determine reliably the weight coefficients for estimating the weighted average value of the frequency of the group measure (standards) based on the results of intercomparisons of measures in the group. Even under the assumption that there is no cross-correlation between the output signals of measures in the group (which contradicts experimental studies), the solution of this system of equations is impossible. Since in this case the rank of the system will be equal to $(N-1)$ in the presence of $N$ unknowns [17]. The rest of the equations of the system in the implementation of the graph of comparisons with the number of nodes exceeding the number $(N-1)$ will be linear combinations of the first $(N-1)$ equations. Resolution of this contradiction requires some subjective assumptions.

Thus, the problem of forming group TS and reference frequencies in the interpretation of the methods of mathematical physics and computational mathematics belongs to the class of ill-posed problems, the solution of which is possible only with the development of an appropriate regularizing algorithm. One of the promising directions in the search for regularizing algorithms is the construction of identification models based on information about the physical processes occurring in the system under consideration [18].

So in the behavior of real group quantum measures (standards), one pattern can be traced: along with the phase drift (stroke of the TS) caused by the deviation of the actual frequency from its nominal value, there are slow phase oscillations relative to its linear drift $[14,15]$. These fluctuations can be classified as a manifestation of the Markov fluctuation process. However, the presence of quasi periodicity of such fluctuations suggests the presence of harmonic components with non-multiple frequencies in the spectrum of the output signal of each measure. Indeed, in the presence of electrical or electromagnetic connections between the QSF (measures) included in the group and located quite close to each other, as well as having electrical connections through the means of mutual comparisons, we can assume the formation of a system of coupled oscillators with close frequencies.

In [14], the presence of regular periodic components in the spectrum of the output signal emitted by the GPS equipment and generated by a group of the QSF on board each satellite is shown. Similar results were obtained in $[15,16]$.

The approach to a group measure as a system of coupled oscillators can allow one to create a model with such a number of parameters that can be unambiguously determined from the results of intercomparisons. For example, the vector equation of state for a group of frequency measures can be represented as a system of differential equations with a set of parameters that are uniquely determined from the results of intercomparisons. This system can be uniquely solved in the class of periodic functions, and the result of the solution can be used to estimate the predicted state of the frequency and phase of the output signal of each measure based on the results of processing their current and previous states. If such estimates are valid, they can be used in the procedures for correcting the output signal of each measure or leading measure in order to compensate for the frequency deviation from the nominal value or the TS stroke.

At present, there are no known hardware methods for compensating the error from interaction in the spectrum of the output signal, since the relative frequency difference between the QSF 
included in the group does not exceed the value $1 \cdot 10^{-11}$. The known methods of numerical processing of results of intercomparisons of the output harmonic signals phases are based on their correlation processing and the subsequent application of the apparatus of the discrete Fourier transform [19]. However, the use of such an approach in the presence of such low frequencies in the spectrum contains two problems.

First, the calculation of the autocorrelation function based on the results of intercomparisons of the QFS included in the group, with a sampling rate equal to one second, requires more than the daily time spent by modern processors.

Second, the autocorrelation function, strictly speaking, is an aperiodic function, and therefore, to determine its spectrum (spectral power density of the phase noise of the QSF), it is necessary to use the integral Fourier transform. In this case, methodically, the apparatus of the discrete Fourier transform is applicable only for strictly periodic functions. Application of this apparatus to aperiodic functions leads to methodological errors in determining the spectral density of the process under study. In addition, the presence of the so-called "frequency masking" effect negates the possibility of accurate and unambiguous determination of the frequency values present in the spectrum of powerful spectral components. Obtaining an estimate of the spectral power density of fluctuations of the phase (frequency) of the QSF output signal is the first step in the mathematical formulation of the problem of constructing the structure of its model. In this case, structural identification is carried out for a qualitative description of the investigated process of fluctuations with the help of various operators.

The basis of the mathematical models describing physical processes in the QSF are most often differential operators. At the same time, a distinction is made between models with lumped parameters, described by ordinary differential equations, and models with distributed parameters, described by partial differential equations. For physical processes taking place in continuous QSF media, the transfer of information about the influencing process occurs through a continuum of material points. In the general case, the variables characterizing the state of the object under consideration (atomic beam, optical cell, etc.) are functions of both time and spatial coordinates. Partial differential equations should be used to describe such a physical process. However, in a number of cases, it is possible to introduce generalizing characteristics or functions into the model, which make it possible to reduce a multidimensional problem to a one-dimensional problem, that is, to go over to a model with lumped parameters.

The second step of the mathematical formulation of the problem is to introduce qualitative information into the QSF model, i.e. to determine (estimate) unknown characteristics (model parameters) included in the structural model. This stage is called parametric identification. Structural and parametric identification of physical processes in the QSF is closely related to the solution of inverse problems for differential equations. When formalizing general formulations and identifying the main classes of inverse problems, it is assumed that the formulations of direct problems are known, each of which can be compared within the framework of an identifiable model with a certain set of inverse problems. In what follows, we will consider physical processes in the QSF from the point of view of "cause - effect" relationships. In accordance with the causal model, causal characteristics include boundary conditions and their parameters, initial conditions, coefficients of differential equations that determine the geometric parameters and material substance of the object under consideration (atomic beam, optical cell, etc.). In addition, the causal characteristics also includes the influence external to the object under consideration, which, as a rule, determines the right side of the differential equations. Then the investigative characteristics will describe the states of the object under study, which are usually understood as fields of physical quantities of one nature or another (temperature field of a quartz resonator, its resonant frequency, phase of the output signal of a measure, etc.).

Causal characteristics do not depend on the investigative manifestations in the sense that the first ones can be specified by rather arbitrary values independently of the second ones.

The selected types of quantities are interconnected by a unidirectional causal relationship, the 
establishment of which is the goal of the direct problem. If, according to certain information about physical fields or processes obtained as a result of measurements, it is required to restore some causal characteristics, then an inverse problem is obtained. So the inverse problems include: the synthesis of the equation of the QSF state, the determination of the impulse or transient characteristics of the object (atomic beam, optical cell, etc.).

Violation of the causal relationship, which takes place in the formulation of the inverse problem, can lead to its mathematical incorrectness [20], most often to the instability of the solution. Therefore, inverse problems represent a typical example of ill-posed problems [21].

By a mathematical model of a certain physical process occurring in the QSF, we mean a set of equations and relations that describe this process, including the initial and boundary conditions for differential equations. In cases where the structure of the QSF mathematical model is given, but some characteristics of the model require their quantitative determination, i.e. it is necessary to solve the problem of parametric identification, in most situations such a problem is solved based on the experimental data (identification of a mathematical model from experimental data). However, another form of identification is possible, e.g., according to the reference mathematical model. In the latter case, the role of the original is played by the process model, which is quite complete and quite accurate, but as a rule, complex and time-consuming in practical application, which necessitates the development of a simpler model. Since the causal characteristics of physical processes in the QSF are usually subject to evaluation, parametric identification is associated with the solution of inverse problems. This leads to the need to determine the correctness of the inverse problem formulation [22]. The Hadamard condition [23] is usually used as a correctness criterion, if the operator equation of the QSF state is obtained

$$
A u=f, u \in U, f \in F,
$$

where $u$ and $f$ are, respectively, the sought and observed characteristics belonging to metric spaces $U, F$, and the operator $A: U \rightarrow F$, which is assumed to be defined by a continuous linear or nonlinear, integral, differential or algebraic operator, has a domain of definition $D(A) \subseteq U$ and a range of values $R(A) \subseteq F . R(A) \subseteq F$.

The problem of solving equation (1) is called correctly posed according to Hadamard if:

1) for any $f \in R(A)=F$ there is a solution $u \in U$ (solvability condition);

2) the solution is unique in $U$ (solvability condition);

3 ) the solution depends continuously on $f$ (stability condition).

If at least one of the listed requirements is violated, problem (1) is called ill-posed.

The question of the existence of a solution to equation (1) consists in the study of belonging $f \in R(A)$. Therefore, $U, F$ spaces should be chosen consistent with each other. For example, if a solution is sought in a specific class of functions, then the choice $f$ cannot be arbitrary, the set of functions on the right-hand side must ensure that the solution to equation (1) belongs to this class.

It is known [24] that most inverse problems of mathematical physics are reduced to solving equations of the first kind (equations of type (1)) with completely continuous compact operators $A$. In this case, $A^{-1}$ operator, inverse of a completely continuous one, is unbounded [23]. As a result of this, the solution of problem (1) with different, but close to each other, right-hand sides $f, f+\tilde{f} \in F$ :

$$
u_{1}=A^{-1} f, u_{2}=A^{-1}(f+\tilde{f}),
$$

can differ from each other as much as desired [25].

Let us consider this situation in somewhat more detail for the case when $A$ operator is given in the form of a linear integral operator generated by a specific type of differential equation in combination with certain initial and boundary conditions: 


$$
A u(x)=\int_{\Omega} u\left(x^{\prime}\right) K\left(x, x^{\prime}\right) d x^{\prime} .
$$

Here the integral is understood in the sense of Lebesgue, $\Omega$ is a measurable region in the $\mathrm{n}$ dimensional space; $K\left(x, x^{\prime}\right)$ is a function measurable in its variables, defined on $\Omega \times \Omega$.

Operator (2) is defined for each measurable function $u(x)$ such that the product $u\left(x^{\prime}\right)$ and $K\left(x, x^{\prime}\right)$ is a function summable over $x^{\prime}$ on $\Omega$ set. Let the function $u(x)$ be treated as an element of space $L_{2}[\Omega]$, and the kernel $K\left(x, x^{\prime}\right)$ satisfies the condition for the existence of a finite integral

$$
\iint_{\Omega} K^{2}\left(x, x^{\prime}\right) d x d x^{\prime}<\infty
$$

It was shown in [25] that such $A$ operator is completely continuous and, therefore, has no bounded converse. Therefore, the values of $A$ operator for arbitrarily large variations in $u(x)$ can differ arbitrarily little from the values of this operator on some $u(x)$ "support" function. As a consequence, the inverse mapping $A^{-1} f$ will not have the property of continuity.

$A$ operator meeting the above requirements is a general case when considering linear formulations of various problems of measuring the QSFparameters. In this case, the kernel $K\left(x, x^{\prime}\right)$ usually corresponds to one or another Green's function or the kernel of the corresponding potential of a simple or double layer [26]. These functions, as a rule, satisfy requirement (3), in particular, they are continuous (a stronger constraint than (3)). Bounded and closed areas are usually considered as $\Omega$.

It is important to note that the transition from problem (1) to its extreme formulation, namely, the search for an element $u$ from the condition of minimizing the residual functional:

$$
u=\arg \inf _{u \in U} \rho_{F}(A u, f),
$$

does not make the task correct.

Residual $\rho_{F}(A u, f)$, as the distance between elements $A u$ and $f$ in space $F$, continuously depends on $f$. Consequently, small changes in $f$ give rise to small changes in the residuals $\rho_{F}(A u, f)$, which, in turn, can correspond to arbitrarily large deviations in the solution and, i.e. convergence in the functional does not imply the convergence of the approximate solutions of the inverse problem to the true one. Moreover, the conditionality of the variational problem (4) as a property characterizing the order of influence of the smallness of the error in the task $f$ on the solution $u$ may turn out to be worse than the conditionality of the original formulation (1).

The correctness of the problem statement from the point of view of the stability of the solution depends on the choice of a pair of spaces $U$ and $F$. This choice cannot be arbitrary. In particular, the right-hand side of equation (1) is usually associated with the results of measurements on some real object and, therefore, is burdened with random errors. These errors occur at any point in the segment $\left[0, \tau_{m}\right]$, i.e. $f_{\delta}(\tau)$ may even be a discontinuous function, which leads to an unstable solution of the inverse problem.

An important role in the solution of inverse problems of measuring the QSF parameters is played by the concept of conditionally correct problems. In [27], requirements are formulated that turn out to be natural in the formulation of problems that are ill-posed in the sense of Hadamard. The essence of these requirements is that an a priori assumption about the existence of a solution and its belonging to a given compact set is added to the conditions of the problem statement. To establish the conditional correctness, it is necessary to prove the uniqueness theorem.

A wide range of studies on conditionally correct problems was carried out in [28 - 30]. Various aspects of the theory of conditionally well-posed problems of mathematical physics are considered 
in [31, 32]. Tikhonov A.N. in [28] introduced the notion of regularization. Its essence is that instead of an unbounded operator giving an exact formula for solving an ill-posed problem, a sequence (regularizing family) of continuous operators is considered such that on each element belonging to the domain of existence of a solution, the corresponding sequence converges to a solution.

One of the interesting approaches to the formulation of problems that are incorrect in the Hadamard sense is the use of concepts and methods of the theory of probability. M.M. Lavrent'ev and V.G. Vasil'ev developed these concepts and methods in the most complete form [32]. In works in this direction, the concept of stability is established, algorithms for solving various classes of problems that are optimal in a certain sense are constructed under certain assumptions about the probabilistic properties of errors in the input data and about the probabilistic properties of the set of sought solutions. In [33], a numerical method was formulated for solving inverse evolutionary equations based on the so-called quasi-inversion. A regularizing operator with a small parameter is added to the evolution equation, which is the product of the original operator and its conjugate one. The small parameter is selected based on the specially developed optimal estimates in the solution. The quasi-inversion method is very simple to implement for solving evolutionary problems of mathematical physics.

The paper [34] presents a method for solving conditionally correct problems of evolutionary type based on the application of the method of minimum residuals for the entire space-time domain of the solution definition. Regularization in this method is performed by choosing the optimal number of steps of the iterative process based on an a priori estimate of the errors in the input data. The trend in the development of methods for solving conditionally correct problems indicates that the methods used are closely related to methods for optimizing the computational process.

\section{Statement of the problem of assessing the instability of passive quantum standards of frequency in the presence of an error from the interaction}

The measurement of the metrological characteristics of the QSF (quantum measure) is carried out by comparing it with a standard or an equally accurate quantum measure using a frequency (phase) comparator. Due to the proximity of the reference frequencies of quantum generators, the comparator cannot provide complete electrical (electromagnetic) isolation of the input signals. As a result, it can be argued about the formation of an electrical (electromagnetic) relationship between the quantum generators involved in the comparison process.

From the sections of the theory of oscillations, it is known that systems of quantum generators having common electrical connections are described by a system of second-order differential equations in the form:

$$
\ddot{U}_{i}+\beta_{i}\left(U_{i}\right) \dot{U}_{i}+\omega_{0 i}^{2} U_{i}=\sum_{\substack{k=1 \\ k \neq i}}^{N} \alpha_{i k} \ddot{U}_{k},
$$

where in $U_{i}=A_{i} \cos \left[\omega_{0 i} t+\psi_{i}(t)\right]$ is electric voltage fluctuations on the output of the QSF with slowly varying amplitudes $A_{i}$ and phases $\psi_{i}(t) ; \beta_{i}\left(U_{i}\right)$ is decrement of the $i$-th quantum generator, a nonlinear function that depends on the signal level of the generator; $\omega_{0 i}$ is the natural resonant frequency of the $i$-th quantum generator; $\omega_{0} \approx \frac{1}{N} \sum_{i}^{N} \omega_{0 i}$ is the nominal value of the frequency of generation of the QFS; $\alpha_{i k}$ is the coupling coefficient between the $i$-th and the $k$-th generators in the group.

System (5) can be reduced using the method of slowly varying amplitudes to a system of truncated first-order differential equations with respect to slowly varying amplitudes and phases of quantum generators included in the system. Under the assumption of the additivity of the noise 
vector $\xi_{i}$, the state vector of system (5) can be written in the form of the differential Langevin equation [17]

$$
\frac{d \vec{\Psi}(t)}{d t}=F[\vec{\Psi}(t), t]+G[\vec{\Psi}(t), t] \vec{\Xi}(t), \vec{\Psi}\left(t_{0}\right)=\vec{\Psi}_{0}
$$

where $\vec{\Psi}(t)=\left\{\Psi_{i}\right\}$ is the $N$-dimensional vector of the current values of the frequency of each quantum measure; $\vec{\Xi}(t)=\left\{\xi_{i}\right\}$ is $N$-dimensional vector of shaping noise (white noise); $F[\vec{\Psi}(t), t]$ and $G[\vec{\Psi}(t), t]$ are deterministic continuously differentiable functions of their arguments that satisfy the Lipschitz condition [35]: $|f(x, y)-f(x, \eta)| \leq M_{b}|y-\eta|, M_{b}$ is some positive constant.

If the functions $F[\vec{\Psi}(t), t]$ and $G[\vec{\Psi}(t), t]$ are known, then it can be argued that the current state of the system of coupled oscillators as a whole has been determined. Indeed, stochastic integration in the sense of Ito [17] of the right and left sides of Eq. (6) (in the extreme case, by numerical methods) leads to an explicit expression of the current state of the system of coupled oscillators $\vec{\Psi}(t)$.

Thus, to solve the problem of determining the metrological characteristics of quantum measures by group comparisons, it is necessary to find in one way or another the expression of the functions $F[\vec{\Psi}(t), t]$ and $G[\vec{\Psi}(t), t]$.

Let us represent the process of measuring phase noise in the form of a mathematical expression, i.e., the observation equation [17]

$$
\vec{Z}(t)=h[\vec{\Psi}(t), t]+\vec{\eta}(t),
$$

where $\vec{Z}(t)$ is the vector of current values of the measurement results, the dimension of which corresponds to the number of meters included in the system; $\left.h^{*}\right]$ the vector-function mathematically expresses the relationship between vectors $\vec{\Psi}(t)$ and measurement results; $\vec{\eta}(t)$ is the noise vector of the meters (comparators).

Since the frequencies of all quantum generators of the system under consideration are very close $\left(\omega_{0} \approx \omega_{0 i} \leq 10^{-10}\right)$, the indicated spectral lines will be in the near zone of the natural frequency of each measure. This will naturally lead to a sharp increase in the power spectral density of the phase noise near the carrier. These spectral lines do not have a frequency multiplicity; therefore, the external manifestations of this phenomenon will be equivalent to the behavior in time of noises such as random wanderings.

Since only the phase difference of the oscillations $\Phi_{i k}=\psi_{i}-\psi_{k}$ generated by the $i$-th and $k$-th measures (QSF) is subject to direct measurement with the help of comparators, in the general case $\psi_{i}$ is not observational. Accordingly, the expression for the single-sideband (SSB) power spectral density of the phase noise, the phase difference $\Phi_{i k}$ in the beat mode will have the form:

$$
S_{\Phi_{i k}}(\omega)=U_{o}^{2} \delta(\omega)+\frac{1}{2} \sum_{k=1}^{K_{i k}} A_{k}^{2} \delta\left(|\omega|-\Omega_{k}\right)+S_{\xi_{i k}}(\omega),
$$

where $\delta(\omega)$ is the symbolic impulse function of Dirac; $S_{\xi_{i k}}(\omega)$ is the power spectral density of the difference in the intrinsic phase noise of the $i$-th and the $k$-th measure; $K_{i k}$ is the number of significant fluctuations in the phase difference between the $i$-th and $k$-th measures, due to beating 
with the signals of other quantum measures in the group and among themselves; $\Omega_{k}$ is the frequency of the $k$-th bright spectral line in the spectrum of the phase of the output signal of the $i$-th measure.

Indirect measurement methods of $S_{\Phi_{i k}}(\omega)$ are based on measuring a set of estimates of the variance of $D_{N}(\tau)$ fluctuations of the phase difference of the output signal of a measure, obtained at different time measurements $\tau$ and subsequent inversion of the Fredholm integral equation of the first kind

$$
2 \int_{0}^{\infty} S_{\Phi_{i k}}(\omega) K_{N}(\omega, \tau) d \omega=D_{N}(\tau)
$$

where $K_{N}(\omega, \tau)$ is the kernel of the integral equation, the form of which is determined by the method of obtaining the estimate $D_{N}(\tau)$ [18].

The inversion of equation (9) by numerical methods is associated with the discretization of this equation and its transformation to a system of algebraic equations of the form:

$$
D_{N}\left(\tau_{i}\right)=\sum_{j=1}^{M} S_{\Phi_{i k}}\left(\omega_{j}\right) K_{N}\left(\omega_{j}, \tau_{i}\right) \Delta \omega, \text { with } i=1,2, \ldots n .
$$

In the matrix form, this expression takes the form:

$$
\vec{D}_{N}=A \vec{S}_{\Phi_{i k}}+\vec{\eta}
$$

where $\vec{D}_{N}^{T}=\left\{D_{N}\left(\tau_{1}\right), \ldots, D_{N}\left(\tau_{N}\right)\right\}$ is the vector of estimates of the variance of the noise of the quantum measure, obtained at the corresponding measurement times of the current frequency value $\tau_{i} ; A$ is a well-known matrix with dimensions $n \times M$, each element of which is multiplied by the quantization step of the original integral equation in the analysis frequency range, and, accordingly, is equal to $\Delta f ; \vec{S}_{\varphi}^{T}=\left\{S_{\varphi}\left(f_{1}\right), \ldots, S_{\varphi}\left(\tau_{M}\right)\right\}$ is the vector of the sought-for values of the spectral power density of the phase noise at the frequencies $f_{i}$.

Matrix $A$ elements represent the numerical values of the square of the modulus of the corresponding frequency response obtained for certain values of the parameters $f=f_{i}$ and $\tau=\tau_{i}$. The variance of the comparator (meter) noise $\sigma_{c}^{2}$, similar to the variance of the quantization noise $\sigma_{q}^{2}$, remains constant for all measurement intervals $\tau_{i}$. Hence, the following expression for the measurement matrix $\Sigma=\left(\sigma_{c}^{2}+\sigma_{q}^{2}\right)$. I is valid, where I is the identity matrix.

The accuracy and stability of solutions is determined by the conditionality of the matrix $\left(A^{T} \Sigma^{-1} A\right)$ [18]. A quantitative estimate of the conditionality of an arbitrary square matrix is the condition number $q=\mu_{\max } / \mu_{\min }$, where $\mu_{\max }$ and $\mu_{\min }$ are the maximum and minimum eigenvalues of the matrix under study. For, $q>10^{v}$ where $v>1$, and not absolutely precisely defined matrix $\left(A^{T} \Sigma^{-1} A\right)$, solution (10) is unstable and has a large error.

Obtaining a stable solution to equation (10) is possible by regularizing it. The essence of the regularization method as applied to the problem under consideration is the transformation of the Fredholm integral equation of the first kind to the Fredholm equation of the second kind [18]. The statistically regularized estimate is in a sense adequate to the Bayesian procedure and comes down to the following. 
Let the a priori (for example, the solution in the form (10)) distribution of the vector $\vec{S}_{\varphi}$ components be known and the first two moments of the random variable $E\left[\vec{S}_{\varphi}\right]=\vec{m}_{S}$ and $E\left[\left(\vec{S}_{\varphi}-\vec{m}_{S}\right)\left(\vec{S}_{\varphi}-\vec{m}_{S}\right)^{T}\right]=P_{S}$ are known. With Gaussian measurement noise $\eta$, the optimal Bayesian estimate $\vec{S}_{\varphi}^{*}$ corresponds to the minimum of the quadratic form $L\left(\vec{S}_{\varphi}\right)=\left(\vec{D}_{N}-A \vec{S}_{\varphi}\right)^{T} \Sigma^{-1}\left(\vec{D}_{N}-A \vec{S}_{\varphi}\right)+\left(\vec{S}_{\varphi}-\vec{m}_{S}\right)^{T} P_{S}^{-1}\left(\vec{S}_{\varphi}-\vec{m}_{S}\right), \quad$ from which, using standard operations, one can obtain a solution to equation (10) in the form:

$$
\vec{S}_{\varphi}^{*}=\left(A^{T} \Sigma^{-1} A+P_{S}\right)^{-1}\left(A^{T} \Sigma^{-1} \vec{D}_{N}+P_{S}^{-1} \vec{m}_{S}\right) .
$$

Thus, the solution of equation (11) makes it possible to evaluate the parameters of the model of interaction of the passive QSF in the process of their comparisons or application in a group standard.

\section{Conclusions}

Based on the results of the analysis of the influence of various external destabilizing factors (ambient temperature, the impact of other QSF) on the characteristics of the output signals of the QSF and methods for their compensation, the following main tasks of further research can be formulated:

1. To develop a method for identifying stochastic processes caused by the error from the interaction of the QSF in a group on the basis of a stochastic model of coupled oscillators, and to develop a method for measuring quantum noise by methods of group standardization.

2. To conduct a study of the behavior of the resonant frequency of a quantum discriminator in a nonstationary fluctuating temperature field of a thermostat and develop a method for compensating for fluctuations in the frequency of a signal generated by a passive QSF caused by fluctuations in the temperature of a thermostat.

3. To develop a method for identifying hidden quasiperiodic processes in the QSF signal using the Fredholm integral equation of the first kind, which connects the noise variance and the power spectral density of phase fluctuations of the output signal containing quasi-harmonic components with non-multiple frequencies.

4. To develop a method for compensating for regular quasiperiodic frequency deviations formed by a group standard based on an identification model of a system of coupled oscillators.

Fundamental experiments in the field of quantum noise in quantum parallel-type random number generators are impossible without precision measurement of time. Therefore, as a promising direction for further research, it is proposed to use the method of identifying a group of quantum standards by a model of a system of coupled oscillators for measuring quantum noise in quantum parallel-type random number generators.

\section{References:}

1. Gorbenko, I.D. and Gorbenko, Yu.I., (2012). Applied cryptology. Theory. Practice. Application, Kharkiv, Ukraine: Fort. 878 p. (in Ukraine).

2. Hofmann-Wellenhof B., Lichtenegger H., \& Wasle E. (2008). GNSS-Global Navigation Satellite Systems GPS, GLONASS, Galileo and more. Vienna: Springer-Velag Wien. 546 p.

3. Peter J.G. Teunissen and O. Montenbruck, (Eds.), Springer Handbook of Global Navigation Satellite Systems, Springer International Publishing, Cham, p. 1335, 2017.

4. Oduan K. Measurement of time. Basics of GPS / Oduan K., Gino B.; Transl. from English. Moscow : Tekhnosfera, 2002. 400 p. (In Russian).

5. Riley, F., (2009) Frequency Standards: Principles and Applications. Moscow : Fizmatlit, 511 p. (in Russian).

6. Audoin C., Guinot B. (2000). The Measurement of Time, Time, Frequency and the Atomic Clock. 346 p. Cambridge University Press. 
7. Schmittberger, B.L., \& Scherer, D.R. (2020). A Review of Contemporary Atomic Frequency Standards. arXiv: Atomic Physics. 18 p.

8. Riley W. J. Handbook of frequency stability analysis. NIST Special Publication 1065. NIST, 2008. 136 pp.

9. M. Hirano, K. Hashimoto, F. Nakagawa, T. Ido, Y. Hanado, and S. Adachi. State estimation for multiple clocks under anomalies using $l_{1}$-norm optimization // Metrologia, vol. 56, no. 2, pp. 1-9, Mar. 2019.

10. C. Zucca and P. Tavella. A mathematical model for the atomic clock error in case of jumps // Metrologia, vol. 52, no. 4, pp. 514-521, June 2015. 285-292.

11. Panfilo G., Harmegnies A., Tisserand L. A new weighting procedure for UTC // Metrologia, 2014, 51(3), p.

12. C. Zucca, P. Tavella (2015). A mathematical model for the atomic clock error in case of jumps. Metrologia. 52. doi: 10.1088/0026-1394/52/4/514.

13. C. Zucca, P. Tavella and G. Peskir (2016). Detecting atomic clock frequency trends using an optimal stopping method // Metrologia. 53. S89-S95. doi: 10.1088/0026-1394/53/3/S89.

14. Trainotti, Christian, Giorgi, Gabriele. Detection and Identification of Phase and Frequency Drifts in Clock Ensembles // Proceedings of the 51st Annual Precise Time and Time Interval Systems and Applications Meeting, San Diego, California, January 2020, pp. 347-365. https://doi.org/10.33012/2020.17310

15. L. Galleani and P. Tavella. Detection and identification of atomic clock anomalies // Metrologia, vol. 45, no. 6, pp. S127-S133, Dec. 2008. 2008.

16. W. J. Riley. Algorithms for frequency jump detection // Metrologia, vol. 45, no. 6, pp. S154-S161, Dec.

17. O. P. Nariezhnii, V. V. Semenets, T. O. Grinenko Method for measuring quantum phase noise and line width of working transition of radio-optical system of random number generator // Telecommunications and Radio Engineering, Volume 77, 2018, Issue 19, pp. 1697-1717. DOI: 10.1615/TelecomRadEng.v77.i19.30

18. Narezhniy A.P. (2005) Identification of latent periodicity in non-stationary phase fluctuations of precision frequency measures // Applied Electronics, 4(2), pp. 148-152 (in Russian).

19. Marple S. L. Digital spectral analysis and its applications. Moscow : Mir, 1990. 584 p. (In Russian).

20. Ivanov V.K. On linear ill-posed problems // DAN USSR, 1962, v. 145, № 2, p. 270 (In Russian).

21. Ivanov V.K. About incorrectly posed tasks // Matematicheskiy sbornik, 1963, v.61, №2, p.211. (In Russian).

22. Ivanov V.K., Vasin V.V., Tanana V.P. The theory of linear ill-posed problems and its applications. Moscow : Nauka Gl. red. fiz.-mat. lit., 1978. 208 p. (In Russian).

23. Lavrent'ev M.M., Romanov V. G., Shishatsky S. P. Incorrect tasks of mathematical physics and analysis. Moscow : Nauka Gl. red. fiz.-mat. lit., 1980. 288 p. (In Russian).

24. Machenov A. S. Solution of linear integral equations of the first kind by the regularization method. Algorithms and programs // VNTICenter, 1975, № 3, p.42 (In Russian).

25. Verlan A. F. Integral equations: Methods, algorithms, programs. Reference book / A. F. Verlan, V. S. Sizikov. K. : Naukova Dumka, 1986. 544 p. (In Russian).

26. Polianin A. D. Handbook of integral equations / Andrei D. Polyanin and Alexander V. Manzhirov. 2nd ed. 2008,1143 p.

27. Tikhonov A. N. Methods for solving ill-posed problems / A. N. Tikhonov, V. Ya. Arsenin. Moscow : Nauka, 1974. 224 p. (In Russian).

28. Tikhonov A. N. On the solution of ill-posed problems and the method of regularization. - DAN USSR. 1963. V. 151. № 3. (In Russian).

29. Lavrentiev M. M. Conditionally well-posed problems for differential equations. Novosibirsk : Izd.-vo NGU, 1973. 224 p. (In Russian).

30. Ivanov V. K., Vasin V. V., Tanana V. P. The theory of linear ill-posed problems and its applications. Moscow : Nauka, 1978. (In Russian).

31. Anikonov Yu. E. Some research methods for multidimensional inverse problems for differential equations. Novosibirsk : Nauka, 1978. (In Russian).

32. Lavrentiev M. M., Vasiliev V. G. On the formulation of some ill-posed problems in mathematical physics // Sib. mat. zh., vol. 7, № 3, 1960. (In Russian).

33. Lyons J., Lattes R. Method of quasi-inversion and its application. Transl. from French. V. O. Sergeeva and V. L. Tsetsokho ; Ed. By M. M. Lavrentiev. Moscow : Mir, 1970. 336 p. (In Russian).

34. Marchuk G. I., Atanbaev S. A. Some questions of global regularization // DAN USSR. 1970. V. 190 . № 3. (In Russian).

35. Ljung L. Systems identification. Theory for the user ; Transl. from English. A. S. Mandel et al. ; Ed. By Ya. Z. Tsypkina. Moscow : Nauka Gl. red. fiz.-mat. lit., 1991. 432 p. (In Russian).

Надійшла до редколегї 09.09.2021 


\section{Відомості про авторів:}

Нарсжній Олексій Павлович - канд. техн. наук, Харківський національний університет імені В.Н. Каразіна, доцент кафедри безпеки інформаційних систем і технологій, факультет комп’ютерних наук, Україна; e-mail: o.nariezhnii@karazin.ua; ORCID: https://orcid.org/0000-0003-4321-0510

Гриненко Тетяна Олексіївна - канд. техн. наук, доцент, Харківський національний університет радіоелектроніки, доцент кафедри безпеки інформаційних технологій, Україна; e-mail: tetiana.grinenko@nure.ua

Горбенко Іван Дмитрович - д-р техн. наук, професор, Харківський національний університет імені В.Н. Каразіна, професор кафедри безпеки інформаційних систем і технологій, факультет комп'ютерних наук, AT «Інститут інформаційних технологій», головний конструктор; Україна; e-mail: GorbenkoI@iit.kharkov.ua; ORCID: https://orcid.org/0000-0003-4616-3449 